\title{
Fast computation of dissipative quantum systems with ensemble rank truncation
}

\author{
Gerard McCaul $\odot,{ }^{1, *}$ Kurt Jacobs $\odot^{2,3}$ and Denys I. Bondar $\oplus^{1, \dagger}$ \\ ${ }^{1}$ Department of Physics, Tulane University, New Orleans, Louisiana 70118, USA \\ ${ }^{2}$ U.S. Army Research Laboratory, Sensors and Electron Devices Directorate, Adelphi, Maryland 20783, USA \\ ${ }^{3}$ Department of Physics, University of Massachusetts at Boston, Boston, Massachusetts 02125, USA
}

(Received 13 October 2020; accepted 10 December 2020; published 8 January 2021)

\begin{abstract}
We introduce a new technique for the simulation of dissipative quantum systems. This method is composed of an approximate decomposition of the Lindblad equation into a Kraus map, from which one can define an ensemble of wave functions. Using principal component analysis, this ensemble can be truncated to a manageable size without sacrificing numerical accuracy. We term this method ensemble rank truncation (ERT), and find that in the regime of weak coupling, this method is able to outperform existing wave-function Monte Carlo methods by an order of magnitude in both accuracy and speed. We also explore the possibility of combining ERT with approximate techniques for simulating large systems [such as matrix product states (MPS)], and show that in many cases this approach will be more efficient than directly expressing the density matrix in its MPS form. We expect the ERT technique to be of practical interest when simulating dissipative systems for quantum information, metrology, and thermodynamics.
\end{abstract}

DOI: 10.1103/PhysRevResearch.3.013017

\section{INTRODUCTION}

In the emerging field of quantum technologies, environments play a crucial role. Their most obvious contribution is to introduce noise which destroys the delicate coherences necessary for truly quantum effects, but system-environment interactions can also harbor some surprising benefits when carefully controlled [1,2]. For example, one may use dissipation to prepare entangled states $[3,4]$, induce nonreciprocal photon transmission [5] and amplification [6], exploit cooperative effects to reduce entropy [7], as well as engineer dynamics $[2,8]$. Given the manifold challenges and opportunities presented by open quantum systems, it is of vital importance to have a fast and scalable method for simulating them.

The first problem one encounters in modeling open systems is how to account for the fact that a full system +environment amalgam exists in a Hilbert space far too large to be simulated exactly. One must instead focus on an effective description of the system of interest, eliminating the environmental degrees of freedom. A number of approaches have been developed to tackle this problem, with one of the earliest examples being the Redfield equation [9-11]. While this equation provides a good approximation to dynamics at weak coupling, it does not guarantee the positivity of the

\footnotetext{
*gmccaul@tulane.edu

$\dagger$ dbondar@tulane.edu
}

Published by the American Physical Society under the terms of the Creative Commons Attribution 4.0 International license. Further distribution of this work must maintain attribution to the author(s) and the published article's title, journal citation, and DOI. density matrix [12], and as such has remained controversial [13].

Another fruitful avenue has been to approach the problem of environmental coupling through the path-integral formalism of the Feynman-Vernon influence functional $[14,15]$. This technique has produced a great number of important results [16-23], including the derivation of a number of distinct dynamical equations, such as the quantum Langevin [24-29], stochastic Schrödinger [30,31], quantum Smoluchowski [32,33], and stochastic Liouville-von Neumann [34-38] equations.

While influence functional techniques (being nonperturbative) are particularly useful at strong environmental coupling, in the weak coupling regime it is far more common to employ the Lindblad master equation [39-41]. This equation represents the most general description for a Markovian process that preserves the essential features of the density matrix (complete positivity and trace preservation) $[42,43]$. It is this generality that has led to the Lindblad equation acquiring its status as the "default" approach to simulating open systems [44], and has found application in a very broad range of physical settings [45-50]. Indeed, it has recently been shown that the secular approximation [51] used to derive the Lindblad equation is unnecessary, giving the equation a much broader regime of applicability than was previously supposed [13,52]. Recent work has also shown that the Lindblad equation can be used for systems undergoing various kinds of broadband control [53].

Given all this, an efficient scheme for computing Lindblad dynamics is of great importance across the many disciplines that use it, enabling the simulation of larger systems which may in turn lead to new discoveries. Achieving this however is problematic, as simulating this master equation requires the density matrix, which for a Hilbert space of size $N_{H}$ has $N_{H}^{2}$ 
elements. This means that simulating the Lindblad equation requires $O\left(N_{H}^{3}\right)$ operations compared to the $O\left(N_{H}^{2}\right)$ operations required to evolve the Schrödinger equation.

Given the exponential increase in $N_{H}$ that occurs as one adds particles to a many-body system, the difference between $O\left(N_{H}^{3}\right)$ and $O\left(N_{H}^{2}\right)$ translates into a tremendous disparity in simulation runtime between the Lindblad and Schrödinger equations for even a moderately large system. While in the last decade enormous strides have been made in the approximate simulation of large systems (see for example recent work simulating strongly interacting quantum thermal machines [54]), the methods employed do not address the fundamental difference in scaling between density matrices and wave functions. Instead, the density matrix is flattened into a vector [55-57] and the power of tensor network decompositions [58] are leveraged to efficiently evolve this large system. In this sense, one has simply transposed the density matrix into a wavefunction living in a Hilbert space of size $N_{H}^{2}$ rather than $N_{H}$. For this reason, there is a powerful motivation to express the Lindblad equation using wave functions of size $N_{H}$ rather than the full density matrix.

The standard solution to this issue has long been to employ a wave-function Monte Carlo (WMC) method (although similar stochastic methods using classical noise have been proposed [59]). This technique exploits the fact that the evolution of the Lindblad equation is equivalent to averaging over an ensemble of stochastic wave-function evolutions [60-65]. If the number of realizations $n_{\text {traj }}$ needed to converge the averaging to an accurate answer is much less than $N_{H}$, then WMC provides an efficient alternative to the exact evolution of the Lindblad equation [66,67]. Of course, like all stochastic methods the number of trajectories needed to converge a WMC result will depend on the specifics of the model system, as well as the length of time being simulated. Consequently, there will be circumstances in which the $n_{\text {traj }}$ required will match or even exceed $N_{H}$, negating the advantages of employing WMC.

In this paper, we introduce a new wave-function based method for describing Lindblad dynamics, which we term ensemble rank truncation (ERT). This method is based on deriving a general expression for the Kraus map of an infinitesimal evolution of the Lindblad equation [up to the second-order error in the time step, $\left.O\left(d t^{2}\right)\right]$. This mapping provides a deterministic procedure for constructing an ensemble of wave functions which together accurately describe system expectations. The size of the ensemble generated through this method increases exponentially with time, necessitating a second procedure to counteract this. Once the size of the ensemble has exceeded a prespecified rank $R$, principal component analysis is performed to generate a truncated set of wave functions in the basis which most efficiently represents the density matrix. This method differs sharply from WMC in two important respects; first, its dynamics are entirely deterministic and do not require any stochastic averaging. This leads to the second distinguishing property, namely that the size of the ensemble produced in this method tends to be much smaller than that required by WMC, with $R \ll n_{\text {traj }}$. Our results show that these features allow ERT to 'beat the house', providing a great improvement over WMC for systems with weak environmental noise (damping and/or dephasing).
A full derivation of our ERT method is presented in Sec. II, together with a formal demonstration that using ERT with matrix product state (MPS) simulations of pure states is more efficient than directly using an MPS representation for a mixed-state evolution. Section III demonstrates the efficacy of ERT in three separate systems - a Heisenberg spin chain, a set of atoms in a cavity, and a dissipative Fermi-Hubbard model. In particular, we show here that ERT can provide order of magnitude improvements in both speed and accuracy simultaneously as compared to the standard WMF approach. We conclude in Sec. IV with a discussion of the results presented here, and suggest potential improvements and extensions to the ERT method.

\section{ENSEMBLE RANK TRUNCATION}

In this section, we derive the approximation we term ensemble rank truncation (ERT). This approximation consists of two parts-an approximate Kraus map which allows the dissipative evolution of the system to be represented with an ensemble of wave functions, and a truncation of that ensemble to its principal components, which is equivalent to excluding components of the density matrix above a certain rank.

\section{A. Approximate Kraus operators}

It is well known that the time evolution of any system (closed or open) must be a completely positive trace preserving (CPTP) map [68], and that such maps on the density matrix can always be decomposed into a Kraus form [69], given by

$$
\hat{\rho}(t)=\sum_{k=1}^{K} \hat{M}_{k} \hat{\rho}(0) \hat{M}_{k}^{\dagger},
$$

where $\hat{\rho}(t)$ is the density matrix and the $\hat{M}_{k}$ are Kraus operators which must collectively satisfy

$$
\sum_{k=1}^{K} \hat{M}_{k}^{\dagger} \hat{M}_{k}=\mathbb{I} .
$$

Expressing a dissipative evolution in this way has obvious advantages compared to the usual master equation, for which the Kraus form may be formally regarded as a solution. Furthermore, if one begins with a pure state $\hat{\rho}(0)=|\psi\rangle\langle\psi|$, it is possible to express the expectation of an operator $\hat{O}$ purely in terms of wave functions, rather than the full density matrix. To do so, one defines a set of wave functions $\left\{\left|\psi_{k}\right\rangle\right\}=$ $\left\{\hat{M}_{k}|\psi\rangle\right\}_{k=1}^{K}$, with which expectations can be expressed as

$$
\langle\hat{O}\rangle=\sum_{k=1}^{K}\left\langle\psi_{k}|\hat{O}| \psi_{k}\right\rangle
$$

The advantage of this form is that rather than an $N_{H} \times N_{H}$ density matrix, one instead requires only $K$ wave functions of dimension $N_{H}$. In the typical case $N_{H} \gg K$, it is far more efficient to calculate expectations via Eq. (3) than with the full density matrix. Of course, this approach is only possible if one knows the $\hat{M}_{k}$ that describe the system of interest.

While there are many existence proofs for Kraus operators describing open system evolutions [70-72] (including for 
controllability of those systems [73]), the number and explicit form of these operators are known only for a few special cases [74]. This is a problem, as one often wishes to solve for the dynamics of a system described by a Lindblad master equation [41], given by

$$
\frac{d \hat{\rho}(t)}{d t}=-\frac{i}{\hbar}[\hat{H}, \hat{\rho}(t)]+\frac{1}{2} \sum_{k=1}^{K}\left(2 \hat{A}_{k} \hat{\rho}(t) \hat{A}_{k}^{\dagger}-\left\{\hat{\rho}(t), \hat{A}_{k}^{\dagger} \hat{A}_{k}\right\}\right),
$$

where $\hat{H}$ is the Hamiltonian and the $\hat{A}_{k}$ are the environmental dissipators. While procedures exist for deriving the Kraus map equivalent to this master equation, deriving the operators is a cumbersome process involving computations that grow exponentially with the number of decay channels [75].

In order to exploit the appealing structure of the Kraus map and avoid the difficulties in calculating the explicit form of the operators, we instead construct an infinitesimal map. To do so, we first discretize Eq. (4):

$$
\begin{aligned}
\hat{\rho}(t+\mathrm{d} t)= & \hat{\rho}(t)-d t \frac{i}{\hbar}[\hat{H}, \hat{\rho}(t)]+O\left(d t^{2}\right) \\
& +\frac{d t}{2} \sum_{k=1}^{K}\left(2 \hat{A}_{k} \hat{\rho}(t) \hat{A}_{k}^{\dagger}-\left\{\hat{\rho}(t), \hat{A}_{k}^{\dagger} \hat{A}_{k}\right\}\right) .
\end{aligned}
$$

It is then possible to represent this expression as a Kraus map:

$$
\hat{\rho}(t+\mathrm{d} t)=\frac{1}{2 K} \sum_{k=1}^{K}\left(\hat{\mathcal{U}}_{k} \hat{\rho}(t) \hat{\mathcal{U}}_{k}^{\dagger}+\hat{\mathcal{V}}_{k} \hat{\rho}(t) \hat{\mathcal{V}}_{k}^{\dagger}\right)+O\left(d t^{2}\right),
$$

where the operators $\hat{\mathcal{U}}_{k}$ and $\hat{\mathcal{V}}_{k}$ are defined as

$$
\begin{aligned}
& \hat{\mathcal{U}}_{k}=\mathrm{e}^{d t \hat{J}_{k}-i \sqrt{K d t} \hat{A}_{k}}, \\
& \hat{\mathcal{V}}_{k}=\mathrm{e}^{d t \hat{J}_{k}+i \sqrt{K d t} \hat{A}_{k}}, \\
& \hat{J}_{k}=-\frac{i}{\hbar} \hat{H}+\frac{K}{2}\left(\hat{A}_{k}^{2}-\hat{A}_{k}^{\dagger} \hat{A}_{k}\right) .
\end{aligned}
$$

Note that in the case of Hermitian dissipators $\hat{A}_{k}^{\dagger}=\hat{A}_{k}$, the Kraus operators are unitary, and the infinitesimal evolution has the form of a random unitary channel [76].

In order to prove Eq. (6), we use the Zassenhaus [77] approximation,

$$
\mathrm{e}^{\Delta(X+Y)}=\mathrm{e}^{\Delta X} \mathrm{e}^{\Delta Y} \mathrm{e}^{-\frac{\Delta^{2}}{2}[X, Y]+O\left(\Delta^{3}\right)},
$$

and after substituting $\Delta=\sqrt{d t}, \quad X=\hat{J}_{k} \sqrt{d t}$ and $Y=$ $\mp i \sqrt{K} \hat{A}_{k}$, we obtain the following representations for the operators to $O\left(d t^{2}\right)$ :

$$
\begin{aligned}
& \hat{\mathcal{U}}_{k}=e^{\hat{J}_{k} d t} U_{k}^{-}+O\left(d t^{2}\right), \quad \hat{\mathcal{V}}_{k}=e^{\hat{J}_{k} d t} U_{k}^{+}+O\left(d t^{2}\right), \\
& \bar{U}_{k}^{ \pm}=e^{ \pm i \hat{A}_{k} \sqrt{K d t}} e^{ \pm d t^{\frac{3}{2}} \frac{\sqrt{K}}{\hbar}\left[\hat{J}_{k}, \hat{A}_{k}\right]} .
\end{aligned}
$$

These approximations can be used to expand a single term in the sum on the RHS of Eq. (6) to $O\left(d t^{2}\right)$ :

$$
\hat{\mathcal{U}}_{k} \hat{\rho}(t) \hat{\mathcal{U}}_{k}^{\dagger}+\hat{\mathcal{V}}_{k} \hat{\rho}(t) \hat{\mathcal{V}}_{k}^{\dagger}=\mathrm{e}^{d t \hat{J}_{k}} \hat{\Gamma}_{k}(t) \mathrm{e}^{d t \hat{J}_{k}^{\dagger}}+O\left(d t^{2}\right),
$$

and after some tedious algebra, one finds

$$
\hat{\Gamma}_{k}(t)=\hat{\rho}(t)+K \mathrm{~d} t\left(\hat{A}_{k} \hat{\rho}(t) \hat{A}_{k}^{\dagger}-\hat{A}_{k}^{2} \hat{\rho}(t)\right)+\text { H.c. }
$$

We now perform a final expansion of $\mathrm{e}^{d t \hat{J}_{k}}$ to $O\left(d t^{2}\right)$ and substitute both this and $\hat{\Gamma}_{k}(t)$ back into Eq. (13) to obtain

$$
\begin{aligned}
\hat{\mathcal{U}}_{k} \hat{\rho}(t) \hat{\mathcal{U}}_{k}^{\dagger}+\hat{\mathcal{V}}_{k} \hat{\rho}(t) \hat{\mathcal{V}}_{k}^{\dagger}=2 \hat{\rho}(t)-2 d t \frac{i}{\hbar}[\hat{H}, \hat{\rho}(t)] \\
+K \mathrm{~d} t\left(2 \hat{A}_{k} \hat{\rho}(t) \hat{A}_{k}^{\dagger}-\left\{\hat{\rho}(t), \hat{A}_{k}^{\dagger} \hat{A}_{k}\right\}\right)+O\left(d t^{2}\right) .
\end{aligned}
$$

Finally, inserting this equality into Eq. (6) recovers Eq. (5) to $O\left(d t^{2}\right)$, proving that $\hat{\mathcal{U}}_{k}$ and $\hat{\mathcal{V}}_{k}$ are the sought-for approximate Kraus operators for an infinitesimal evolution. Note also that these operators approximately satisfy Eq. (2) with

$$
\frac{1}{2 K} \sum_{k=1}^{K}\left(\hat{\mathcal{U}}_{k}^{\dagger} \hat{\mathcal{U}}_{k}+\hat{\mathcal{V}}_{k}^{\dagger} \hat{\mathcal{V}}_{k}\right)=\mathbb{I}+O\left(d t^{2}\right)
$$

\section{B. Truncating the ensemble}

For a single time step, the computational complexity of performing the matrix-matrix calculation for the exact Lindblad equation is $O\left(N_{H}^{3}\right)$, but employing the approximate Kraus form, obtaining the set of wave functions $\left\{\left|\psi_{k}\right\rangle\right\}=$ $\left\{\hat{\mathcal{U}}_{k}|\psi\rangle, \hat{\mathcal{V}}_{k}|\psi\rangle\right\}_{k=1}^{K}$ needed to calculate expectations has a computational complexity of only $O\left(K N_{H}^{2}\right)$. Furthermore, using the approximate method one need only store $K N_{H}$ elements, whereas the density matrix requires $N_{H}^{2}$ elements. Clearly for a single step, if $N_{H} \gg K$, both the speed and storage requirements for the calculation will be improved by approximately a factor of $N_{H}$. Of course, the major problem with this method is that after each step, the number of wave functions increases by a factor of $2 K$, so that after $M$ steps one must store a set of $(2 K)^{M}$ wave functions.

To overcome this bottleneck, we employ a further approximation, aimed at limiting the size of the wave-function ensemble. First, let $R$ denote the prespecified maximal rank of the density matrix $\hat{\rho}$. The choice of $R$ is dictated by physics, the desired accuracy, and the available memory. For simplicity we assume that the initial density matrix is represented by

$$
\hat{\rho}(t)=|\psi\rangle\langle\psi|,
$$

although the generalization to a mixed density matrix is trivial. The procedure is then as follows: we propagate according to Eq. (6), saving the set $\left\{\left|\psi_{k}\right\rangle\right\}$ at each step until there are $L=$ $(2 K)^{M}>R$ wave functions. Now the size of this set exceeds $R$, we orthogonalize them such that

$$
\hat{\rho}(t+M d t)=\sum_{k=1}^{L}\left|\psi_{k}\right\rangle\left\langle\psi_{k}\left|=\sum_{k=1}^{L}\right| \bar{\psi}_{k}\right\rangle\left\langle\bar{\psi}_{k}\right|
$$

with $\left\{\left|\bar{\psi}_{k}\right\rangle\right\}$ being orthogonal but unnormalized. This orthogonalization is achieved by

$$
\left|\bar{\psi}_{k}\right\rangle=\sum_{l=1}^{L} U_{l k}\left|\psi_{l}\right\rangle
$$

where $U$ is a unitary matrix obtained from diagonalizing the overlap matrix $S_{i j}=\left\langle\psi_{i} \mid \psi_{j}\right\rangle$, such that

$$
U^{\dagger} S U=\operatorname{diag}\left(w_{1}, \ldots, w_{L}\right) .
$$

Here we assume that the eigenvalues are arranged in descending order, $w_{1} \geqslant \cdots \geqslant w_{L}$. Note that obtaining the overlap matrix involves $L^{2}$ wave-function dot-products, meaning its 


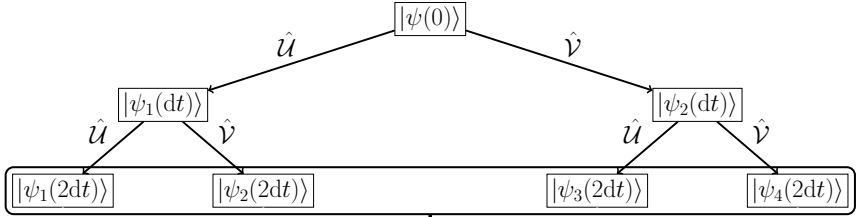

$\left.\langle\hat{O}\rangle=\sum_{k=1}^{R}\left\langle\bar{\psi}_{k}^{R}|\hat{O}| \bar{\psi}_{k}^{R}\right\rangle|| \bar{\psi}_{k}^{R}\right\rangle=\sum_{l=1}^{L} U_{l k}^{R}\left|\psi_{l}\right\rangle$
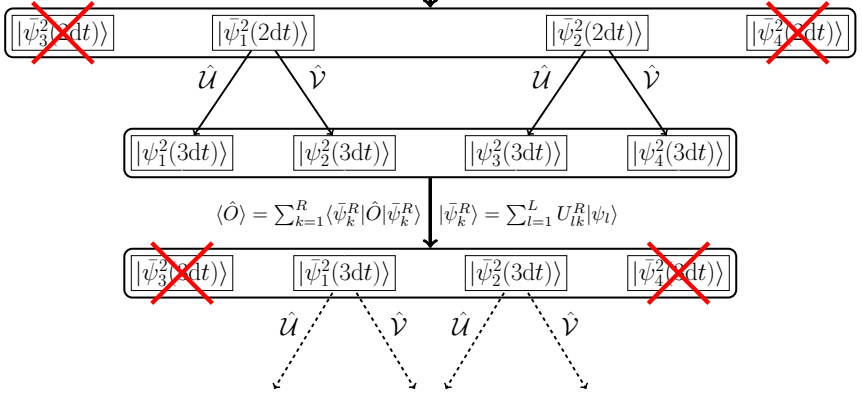

FIG. 1. Schematic showing the ERT process for a single dissipator and $R=2$. The initial wave function is propagated by the application of the $\mathcal{U}$ and $\mathcal{V}$ unitaries until the size of the set exceeds $R$. Each time this occurs the orthogonalization procedure is performed and the $R$ largest components of the set are retained for propagation to later steps.

computational cost is $O\left(L^{2} N_{H}\right)$. For $N_{H} \gg L^{2}$, this has a negligible cost relative to other operations. Furthermore, since the dimensionality of matrix $S$ is $L \times L$, its diagonalization is also computationally "cheap" and does not depend on the dimensionality $N_{H}$ of the Hilbert space.

Having calculated $U$, we can truncate it to an $R \times L$ matrix $U^{R}$, using the eigenvectors associated with the $R$ largest eigenvalues. $U^{R}$ can then be used to generate an orthogonal ensemble of wave functions:

$$
\left|\bar{\psi}_{k}^{R}\right\rangle=\sum_{l=1}^{L} U_{l k}^{R}\left|\psi_{l}\right\rangle .
$$

The generation of this truncated ensemble is akin to principal component analysis, a well-known statistical technique where $R$ is the number of principal axes [78]. Using this procedure we need only store $\left\{\left|\bar{\psi}_{k}^{R}\right\rangle\right\}_{k=1}^{R}$, and after appropriate normalization of this set we obtain the sought for ERT approximation,

$$
\hat{\rho}(t+M d t) \approx \sum_{k=1}^{R}\left|\bar{\psi}_{k}^{R}\right\rangle\left\langle\bar{\psi}_{k}^{R}\right| .
$$

Propagating to later steps then simply repeats the same process of orthogonalization followed by truncation to $R$ wave functions, as shown in Fig. 1. Finally, we emphasize that stepping forward in time by generating the set $\left\{\hat{\mathcal{U}}_{m}\left|\bar{\psi}_{k}^{R}\right\rangle, \hat{\mathcal{V}}_{m}\left|\bar{\psi}_{k}^{R}\right\rangle\right\}_{m=1}^{K}$ is a fully parellelizable calculation, so that if $2 K R$ does not exceed the number of threads available, the time required for stepping forward will be approximately independent of the rank. Furthermore, in the case of large systems where the calculation of matrix exponents is prohibitively costly, the effect of applying any given Kraus operator to a member of the ensemble can instead be characterized as an ODE (with an explicit time step of $\sqrt{d t}$ ). A repeated propagation via Eq. (6) can also be represented as a noncommutative Newton binomial $[79,80]$. This insight offers a potential route to speed up calculations.

\section{Combining ensemble rank truncation with matrix product states}

It is worth taking a moment to place the ERT method in its proper context. It is the product of two separate approximations-an approximation for the infinitesimal Kraus operators which allows one to characterize the dynamics with a set of wave functions, and a truncation of that set to its principal components at each time step. The former approximation is to the best of our knowledge a novel representation of the density matrix evolution, while the reduction of the set of wave functions to their principal components is designed to constrain the size of the ensemble to a manageable size.

It is interesting to note that both ERT and the matrixproduct-state (MPS) representation [81-83] make use of a singular-value decomposition to find the most efficient representation for a state. In the case of MPS, it is used to find minimal-rank Schmidt decompositions to minimize the matrices that form the MPS representation for a pure state. Here it is used to minimize the number of pure states that are required to represent a mixed state.

In fact, there is no reason why ERT cannot be performed on sets of wave functions evolved using time-dependent MPS (time evolving block decimation (TEBD) [82]). MPS has previously been combined with stochastic wave-function methods to simulate spin chains in a cavity [84], and one might ask whether combining ERT with an MPS representation for pure states provides any advantage versus directly using MPS to represent the evolving vectorized density matrix. To answer this, recall that in the MPS representation $[81,82]$, a quantum state $|\Psi\rangle$ is written as

$$
\begin{aligned}
|\Psi\rangle & =\sum_{i_{1}=0}^{d-1} \cdots \sum_{i_{n}=0}^{d-1} c_{i_{1} \cdots i_{n}}\left|i_{1}\right\rangle \otimes \cdots \otimes\left|i_{n}\right\rangle, \\
c_{i_{1} \cdots i_{n}} & =\sum_{\alpha_{1}, \ldots, \alpha_{n-1}=1}^{\chi} \Gamma_{\alpha_{1}}^{[1] i_{1}} \lambda_{\alpha_{1}}^{[1]} \Gamma_{\alpha_{1} \alpha_{2}}^{[2] i_{2}} \lambda_{\alpha_{1}}^{[2]} \cdots \Gamma_{\alpha_{n-1}}^{[n] i_{n}},
\end{aligned}
$$

where $\left\{\left|i_{1}\right\rangle \otimes \cdots \otimes\left|i_{n}\right\rangle\right\}$ is a computational basis for an $n$ body system, and $\chi$ is the Schmidt rank quantifying the degree of entanglement. In other words, a state $|\Psi\rangle$ is represented by $n$ tensors $\Gamma^{[1]}, \ldots, \Gamma^{[n]}$ (each with $\chi d$ elements) and $n-1$ length $\chi$ vectors $\lambda^{[1]}, \ldots, \lambda^{[n-1]}$.

We may apply the MPS representation directly to a density matrix $\hat{\rho}$ that has been flattened into a column vector $|\hat{\rho}\rangle_{\sharp}[56]$. Using Eq. (23) the vectorized density matrix is expressed as

$$
\begin{aligned}
|\hat{\rho}\rangle_{\sharp} & =\sum_{j_{1}=0}^{d^{2}-1} \cdots \sum_{j_{n}=0}^{d^{2}-1} c_{j_{1} \cdots j_{n}}^{\sharp}\left|j_{1}\right\rangle_{\sharp} \otimes \cdots \otimes\left|j_{n}\right\rangle_{\sharp}, \\
c_{j_{1} \cdots j_{n}}^{\sharp} & =\sum_{\alpha_{1}, \ldots, \alpha_{n-1}=1}^{\chi} \Gamma_{\sharp \alpha_{1}}^{[1] j_{1}} \lambda_{\sharp \alpha_{1}}^{[1]} \Gamma_{\sharp \alpha_{1} \alpha_{2}}^{[2] j_{\alpha_{1}}} \lambda_{\alpha_{1}}^{[2]} \cdots \Gamma_{\sharp \alpha_{n-1}}^{[n] j_{n}},
\end{aligned}
$$


where $\left\{\left|j_{n}\right\rangle_{\sharp}\right\}_{j=0}^{d^{2}-1}$ is a set of the vectorized matrices $\left\{\left|k_{n}\right\rangle\left\langle l_{n}\right|\right\}_{k, l=0}^{d-1}$. In this case, the tensors $\Gamma_{\sharp}^{[k]}$ will each be composed of $\chi d^{2}$ elements. Consequently the vectorized density matrix in Eq. (24) will possess $n d$ times more parameters than the wave function in Eq. (23). It therefore follows that so long as $2 K R<n d$, it will be more efficient to capture the system behavior using ERT to construct an ensemble of R MPS wave functions rather than evolving a single vectorized density matrix with MPS. The reason for this boost is that with the help of principle component analysis ERT finds the optimal basis to represent a given density matrix; whereas, MPS explores the sparsity of the vectorized density matrix. Of course, by combining ERT with MPS, one must now work under the double assumption that the system has both low rank and low entanglement.

\section{SIMULATION RESULTS}

We now demonstrate the utility of ERT over WMC by applying it to two typical multi-body systems of enduring interest (a Heisenberg spin-chain and a collection of two-level systems coupled to a cavity mode). We choose these systems to be small enough that the Linblad equation can be simulated directly for comparison, but large enough that the ensemble methods are significantly faster.

We also provide an example of applying ERT to fermionic systems by calculating the power spectrum generated by a driven dissipative Fermi-Hubbard system. The convergence of ERT to the exact result is first checked at a smaller system size, before being applied to a system too large to practically calculate (on the hardware used) the exact dynamics.

In order to assess the performance of the method we introduce an integrated error $\mathcal{E}$ for a set of observables $\left\{\hat{O}_{j}\right\}$ :

$$
\mathcal{E}=\left(\sum_{j} \frac{\int d t\left(O_{j}(t)-O_{j}^{A}(t)\right)^{2}}{\int d t O_{j}^{2}(t)}\right)^{1 / 2},
$$

where $O_{j}(t)$ is the observable expectation from the exact simulation and $O_{j}^{A}(t)$ is the expectation calculated using an approximate method. In the example simulations considered, when the integrated error is on the order of $10^{-1}$ the accuracy is more than adequate for most purposes, and an error of $10^{-2}$ represents an excellent reproduction of the exact dynamics.

\section{A. Heisenberg spin chain}

We first consider a simple $N$ site spin-chain system, specifically a Heisenberg XXX model described by the Hamiltonian:

$$
\hat{H}=-\pi h \sum_{j=1}^{j=N} \hat{\sigma}_{z}^{(j)}-\pi J \sum_{j=1}^{j=N-1} \hat{\boldsymbol{\sigma}}^{(j)} \cdot \hat{\boldsymbol{\sigma}}^{(j+1)} .
$$

Historically Hamiltonians of this type have been used to model magnetic systems [85] in order to calculate their critical points and phase transitions [86]. More recently, this class of models has also been used to study exotic phenomena such as many-body localization [87].

Given that our principal interest in this model is to assess the performance of ERT, in all cases, we set $h=J=1$, and initialize the system with all spins in the $+x$ direction
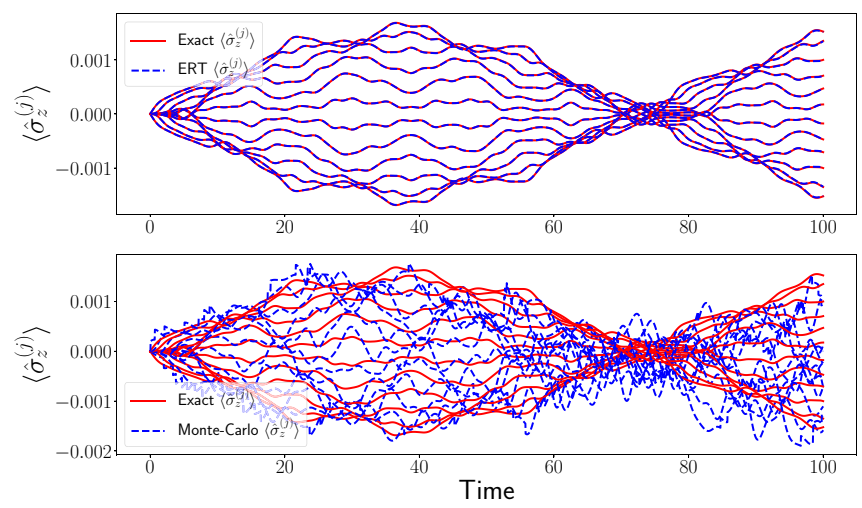

FIG. 2. Dynamics of $\left\langle\hat{\sigma}_{z}^{(j)}\right\rangle$ (without explicit labeling for individual sites) for $\gamma=0, \Gamma=10^{-3} h$, and $\mu=0.9$. The top panel shows the rank 1 approximation, which runs in 159 seconds with an integrated error $\mathcal{E}=1.3 \times 10^{-2}$. In comparison, a WMC simulation (shown in the bottom panel) with 1000 trajectories sampled runs in 4925 seconds with an integrated error of $\mathcal{E}=3.3$. The exact dynamics runs in 9790 seconds, demonstrating that in this weak environmental coupling regime, ERT significantly outperforms the WMC method in both accuracy and efficiency.

(i.e., $\left\langle\hat{\sigma}_{x}^{(j)}\right\rangle=1$ ). We choose $N=12$ sites corresponding to a Hilbert space dimension of $N_{H}=2^{12}=4096$.

In addition to the dynamics of the chain itself, we shall include two types of dissipator: an onsite dephasing

$$
\hat{A}_{j}=\sqrt{\gamma} \hat{\sigma}_{z}^{(j)},
$$

and a set of spin injection/absorption operators at the chain terminals:

$$
\hat{A}_{1,2}^{\prime}=\sqrt{\Gamma(1 \mp \mu)} \hat{\sigma}_{ \pm}^{(1)}, \quad \hat{A}_{3,4}^{\prime}=\sqrt{\Gamma(1 \pm \mu)} \hat{\sigma}_{ \pm}^{(N)} .
$$

For both types of dissipator, $\gamma$ and $\Gamma$ characterize the strength of the environmental coupling to the spin system. For the latter, $\mu$ biases the driving (analogously to a chemical potential). Lindblad master equations are only appropriate when the damping is sufficiently slow compared to the internal dynamics. We explore the performance of ERT for damping rates $\Gamma \in\left[10^{-3} h, 10^{-1} h\right]$, which covers essentially the full range of dissipation strengths (relative to the size of the Hamiltonian couplings) over which Lindblad equations are of interest. We explore the behavior both without dephasing and with a weak dephasing rate of $\gamma=10^{-3} \mathrm{~h}$. When evaluating the simulation error using Eq. (25), we use the set of observables $\hat{\sigma}_{x}^{(j)}, \hat{\sigma}_{z}^{(j)}$.

The numerical simulations of this spin chain are performed using the Quantum toolbox in PYTHON (QUTIP) [88,89], which includes optimized implementations of both the exact Lindblad master equation, and a parallelized WMC approximation. All simulations are performed using 4 cores of an Intel Xeon 8280 CPU (with eight hardware threads), and the ERT method is implemented without any explicit parallelization. Despite this, Figs. 2 and 3, show that even an unoptimized PYTHON implementation of ERT is able to perfectly reproduce the exact Lindbladian dynamics, improving upon the exact simulation runtime by almost two orders of magnitude. More significantly, the same ERT implementation also significantly outperforms the optimized C implementation of the QUTIP WMC code in both accuracy and runtime. 

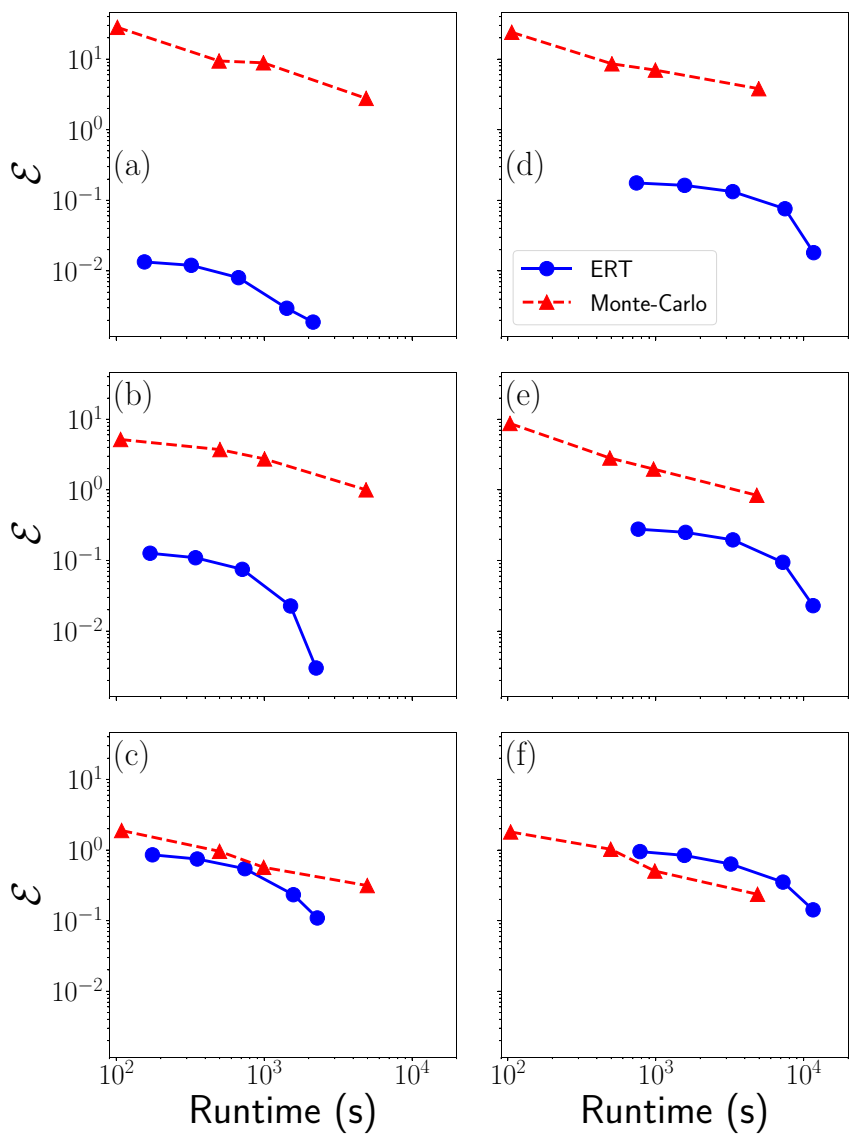

FIG. 3. Comparison of spin-chain simulation accuracy to runtime for both WMC and ERTs over a variety of dissipative parameters, using $\mu=0.9$. For (a)-(c), $\gamma=0$, while (d)-(f) use $\gamma=10^{-3} h$. For (a) and (d), $\Gamma=10^{-3} h$, (b) and (e) $\Gamma=10^{-2} h$, and finally (c) and (f), $\Gamma=10^{-1} h$. Points are generated using the set $R=\{1,2,4,8,12\}$ for ERT, and $n_{\text {traj }}=\{100,500,1000,5000\}$ trajectories for the WMC simulations. Exact simulations run in $\sim 9500$ seconds on average.

A more systematic comparison of results is shown in Fig. 3, demonstrating the relative performance of the low-rank and WMC methods both with and without dephasing, while varying $\Gamma$ between $\Gamma=10^{-3} h$ and $10^{-1} h$.

The first conclusion that can be drawn is that ERT yields larger advantages at weaker couplings. This fits with the natural intuition that at weaker couplings the set of wave functions generated by the unitaries in Eq. (6) will be well characterized by a relatively small set of orthogonal components. Nevertheless, even at the strongest damping considered, ERT offers comparable accuracy to WMC. In the case of nonzero dephasing, the presence of an additional 12 dissipators reduces the relative accuracy of ERT at lower ranks (although it remains more accurate than the equivalent WMC simulation for the majority of points). Significantly, runtimes are increased by the presence of additional dissipators, but this is to be expected when the calculation of the new wave functions at each time step is run serially rather than in parallel. In this instance, the runtime for a single time step will be roughly proportional to $2 K R$, as one must apply each of the $2 K$ unitaries to the set of $R$ wave functions. Since each of these operations is in- dependent however, significant efficiencies could be achieved by parallelising this process when more cores are available.

\section{B. Ensemble of two-level systems in a cavity}

Our second example is a hybrid system consisting of a single cavity mode coupled to a number of otherwise independent two-level systems (which represent, e.g., cold atoms [90], color centers [91], or rare-earth dopants [92]). We assume that all the emitters are coupled to the same zero-temperature bath, and the cavity is coupled to a separate input/output port (e.g., a lossy end mirror).

In a recent development, a master equation in the Lindblad form has been obtained that accurately describes open systems regardless of the proximity (or otherwise) of the various transitions (and that is valid for all temperatures) [13]. Employing this master equation, placing the two-level systems on resonance with the cavity mode and working in the interaction picture, the effective system Hamiltonian is given by

$$
\begin{aligned}
\hat{H}= & \sum_{i j} \hat{\sigma}_{+}^{(j)} \Lambda_{i j} \hat{\sigma}_{-}^{(j)}+g \sum_{j}\left(\hat{a}^{\dagger} \hat{\sigma}_{-}^{(j)}+a \hat{\sigma}_{+}^{(j)}\right) \\
& +\sqrt{\kappa} \beta\left(\hat{a}+\hat{a}^{\dagger}\right) .
\end{aligned}
$$

Here $\Lambda_{i j}=\Delta_{i} \delta_{i j}+\sqrt{\lambda_{i} \lambda_{j}}$ includes the detuning between emitter $i$ and the cavity, $\Delta_{i}$, as well as the respective bathinduced Lamb shifts, $\lambda_{i}$. Here $\hat{\sigma}_{-}^{(j)}$ is the lowering operator for the $j$ th atom from its excited to its ground state and $\hat{a}$ is the annihilation for the cavity mode. The term proportional to $g$ is the coupling between the emitters and the cavity mode, and the last term describes driving of the cavity with a coherent state at the rate of $|\beta|^{2}$ photons per second [93]. The Lindblad dissipators for the system are given by

$$
\hat{A}_{1}=\sqrt{\kappa} \hat{a}, \quad \hat{A}_{2}=\sqrt{\gamma} \sum_{j} \hat{\sigma}_{-}^{(j)},
$$

which describes output coupling of the cavity mode at rate $\kappa$ and collective decay of the exited states of all the emitters at the rate $\gamma$.

Once again we focus on the regime of weak coupling, setting $g=1, \Lambda_{i j}=20 j g \delta_{i j}$, and $\kappa=\beta$ For all simulations we take eight atoms and initialize each atom in an equal superposition of its ground and excited states, along with an empty cavity. Simulations are run with the same resources as in the previous section, while the simulation error Eq. (25), uses the observables $\hat{\sigma}_{x}^{(j)}, \hat{\sigma}_{z}^{(j)}$ and $\hat{a}^{\dagger} a$. As Fig. 4 demonstrates, ERT is again able to outperform WMC in capturing the decay of the atomic ensemble at a smaller computational cost. When varying $\gamma$ and $\kappa$, Fig. 5 shows once again that for weak couplings ERT provides a clear advantage over WMC in both efficiency and accuracy. Even at the largest $\gamma$ considered, we see that at higher ranks the performance of ERT becomes comparable to WMC. This trend is repeated both when including off-diagonal elements in $\Lambda_{i j}$, and initialising the system from different states (not shown).

\section{Dissipative Fermi-Hubbard model}

Finally we apply the ERT method to a fermionic system, specifically the Fermi-Hubbard model [94]. This model is a 


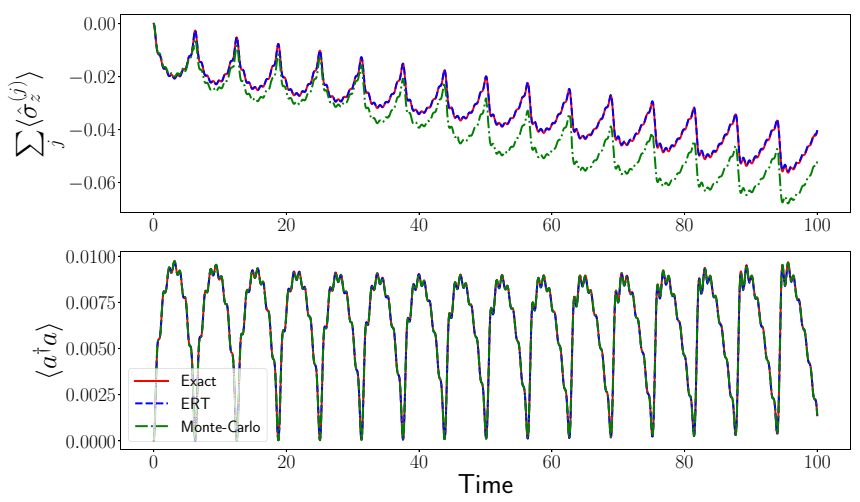

FIG. 4. Dynamics of the atomic ensemble in a cavity for $\gamma=$ $10^{-3} \mathrm{~g}$ and $\beta=\kappa=10^{-1} \mathrm{~g}$, with the exact, rank $1 \mathrm{ERT}$, and 1000 trajectory WMC dynamics shown. The top panel shows the collective atomic dynamics while the bottom panel demonstrates the cavity occupation numbers. The exact simulation takes 5975 seconds to compute, while the WMC simulation runs in 736 seconds, with an integrated error over all expectations of $\mathcal{E}=0.3$. In comparison the ERT computation completes in 32 seconds with an integrated error of $\mathcal{E}=2.0 \times 10^{-2}$, improving on WMC by over an order magnitude in both accuracy and runtime.

useful benchmark, given its status as one of the paradigmatic models for strongly correlated electronic systems. Its Hamiltonian is given by

$$
\begin{aligned}
\hat{H}(t)= & -t_{0} \sum_{j \sigma}\left(\hat{c}_{j \sigma}^{\dagger} \hat{c}_{j+1 \sigma}+\hat{c}_{j+1 \sigma}^{\dagger} \hat{c}_{j \sigma}\right) \\
& +U \sum_{j} \hat{c}_{j \uparrow}^{\dagger} \hat{c}_{j \uparrow} \hat{c}_{j \downarrow}^{\dagger} \hat{c}_{j \downarrow},
\end{aligned}
$$

where $\hat{c}_{j \sigma}$ is the fermionic annihilation operator for the $j$ th site and spin $\sigma, t_{0}$ is the hopping parameter and $U$ is the onsite potential. The Fermi-Hubbard model is of particular interest as under coherent external driving it exhibits a high degree of complexity in its dynamics $[95,96]$. When dissipation is added this model contains a number of intriguing features, including symmetry breaking phase transitions [97] and dynamic reversals of magnetic correlations [98].

To incorporate dissipation, we apply fermion injection/absorption operators for each species to the terminals of an $N$ site chain

$$
\begin{array}{ll}
\hat{A}_{1,2}^{\prime}=\sqrt{\Gamma(1-\mu)} \hat{c}_{1\{\uparrow, \downarrow\}}, & \hat{A}_{3,4}^{\prime}=\sqrt{\Gamma(1+\mu)} \hat{c}_{1\{\uparrow, \downarrow\}}^{\dagger}, \\
\hat{A}_{5,6}^{\prime}=\sqrt{\Gamma(1+\mu)} \hat{c}_{N\{\uparrow, \downarrow\}}, & \hat{A}_{7,8}^{\prime}=\sqrt{\Gamma(1-\mu)} \hat{c}_{N\{\uparrow, \downarrow\}}^{\dagger}
\end{array}
$$

and use these dissipators to drive the system from its (isolated) half-filled ground state.

In order to simulate this model, we use the PYTHON package QUSPIN [99,100], which is capable of efficiently simulating fermionic systems. Note that as QUSPIN lacks an efficient WMC solver for dissipative systems, in this example we forgo comparison to approximate methods.
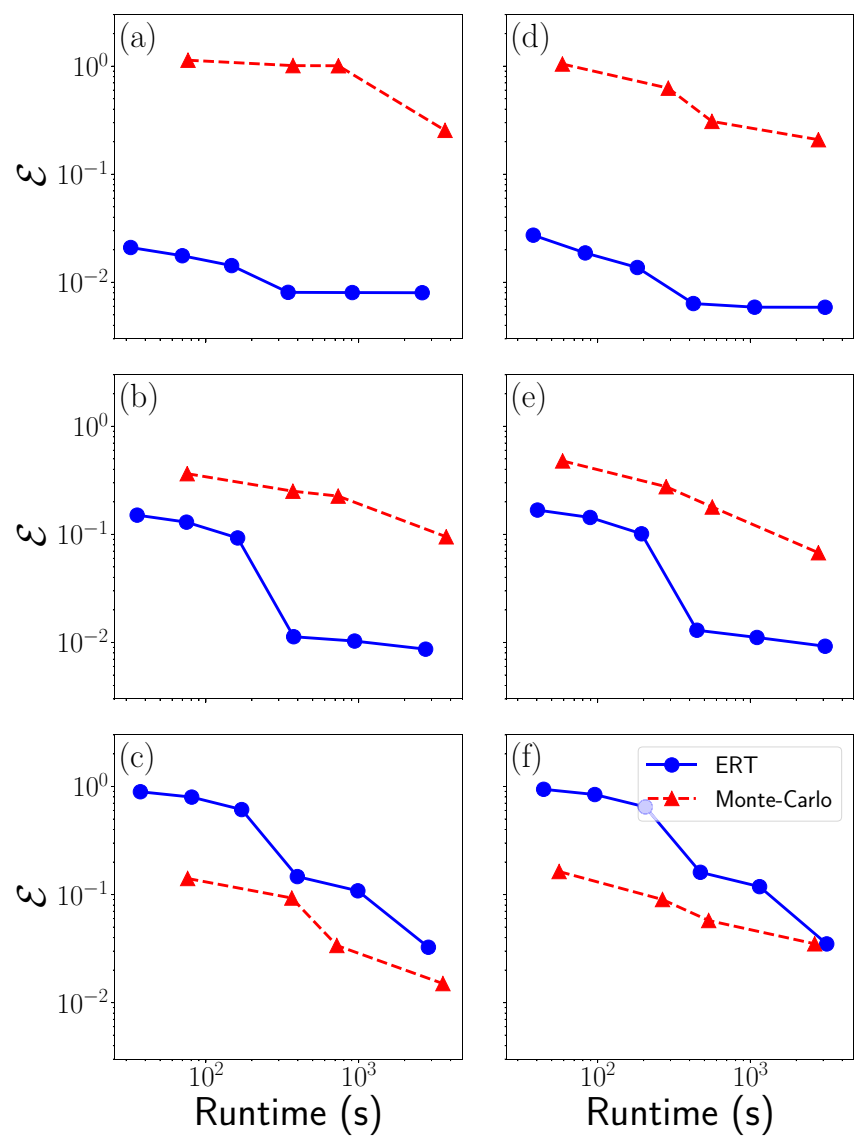

FIG. 5. Accuracy vs runtime results for the two-level systems in a cavity over a variety of dissipative parameters. For (a)-(c), $\kappa=$ $10^{-1} g$, while (d)-(f), use $\kappa=g$. For (a) and (d), $\gamma=10^{-3} g$, (b) and (e), $\gamma=10^{-2} g$, and finally (c) and (f), $\gamma=10^{-1} g$. Once again we see that ERT provides a decisive advantage at weak couplings, but requires higher ranks to properly capture dynamics at stronger couplings. Points are generated using the set $R=\{1,2,4,8,16,32\}$ for ERT, and $n_{\text {traj }}=\{100,500,1000,5000\}$ trajectories for the WMC simulations. Exact simulations run in $\sim 5500$ seconds on average.

In the case of a coherently driven, nondissipative system, the electronic current

$$
\hat{J}(t)=-t_{0} \sum_{j \sigma}\left(\hat{c}_{j \sigma}^{\dagger} \hat{c}_{j+1 \sigma}-\hat{c}_{j+1 \sigma}^{\dagger} \hat{c}_{j \sigma}\right)
$$

may exhibit an optical phenomenon known as high harmonic generation (HHG). HHG occurs when the dipole acceleration $a(t)=\frac{d J(t)}{d t}$ spectrum has a a highly nonlinear response to driving, generating frequencies many multiples larger than the driving field [101-103]. It is therefore natural to ask if similar nonlinearities are present when one uses incoherent dissipative driving. As an initial test of the applicability of ERT to this model, we calculate the dipole acceleration $a(t)$ in a $U=t_{0}, N=6\left(N_{H}=4^{6}=4096\right)$ system and compare it to the results of an exact simulation. As Fig. 6 shows, once again ERT is able to obtain good accuracy while still significantly improving on the time taken to run the calculation via exact methods.

Using ERT we are able to investigate whether or not nonlinear effects are present in this dissipatively driven model 

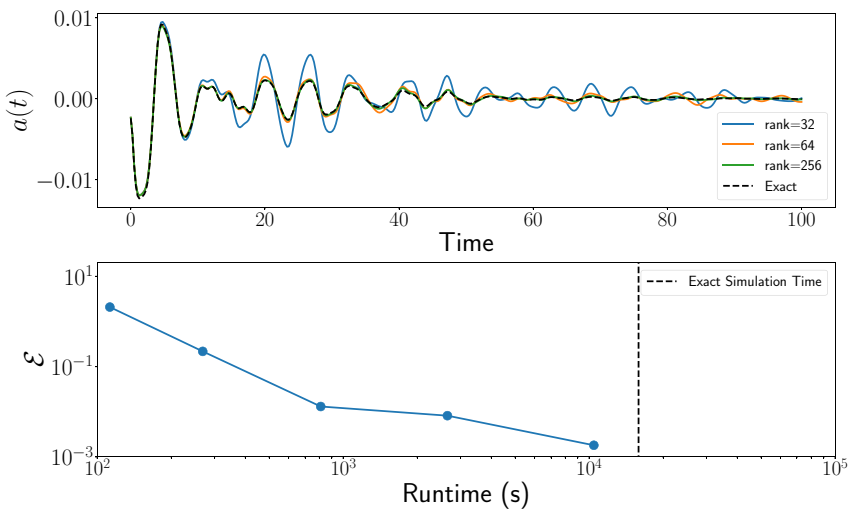

FIG. 6. Calculation of the dipole acceleration $a(t)=\frac{d J(t)}{d t}$ in a $U=t_{0}, N=6$ site system at a relatively strong coupling $\Gamma=0.03 t_{0}$ and bias $\mu=0.9$. The top panel shows the time dynamics of $a(t)$, while the bottom panel demonstrates the relative accuracy and efficiency of the approximation using $R=\{16,32,64,128,256\}$.

at larger system sizes than can be practically calculated using exact methods. As an example, we consider the $U=0$, $N=14$ Fermi-Hubbard model with dissipative driving. In this free case, Hamiltonian symmetries reduce the effective size of the Hilbert space to $N_{H}=16384$, but this is still well beyond the dimension at which an exact master equation calculation is practical without significant computational resources. One can test the accuracy of the approximation in this scenario in a number of ways - the most straightforward being to check that as the rank $R$ is increased, simulation results converge. A second check in this instance is utilising the fact that the Hubbard model is known to exhibit diffusive transport when dissipatively driven [104]. From this, one would expect that the steady state currents $J_{f}$ should depend linearly on the coupling strength. Figure 7 shows that at sufficiently large ranks this is the case, providing a further check for the accuracy of ERT.
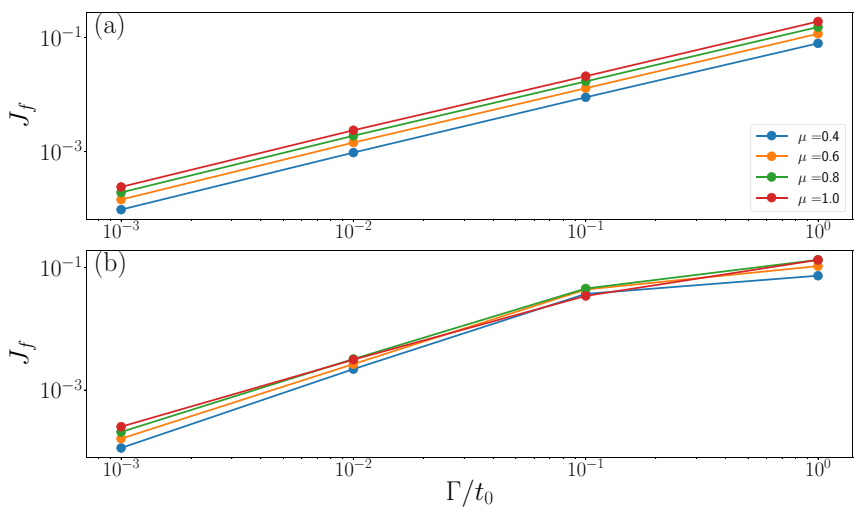

FIG. 7. Plotting steady state currents $J_{f}$ against coupling strength, using (a) $R=128$ and (b) 16. One finds that at higher ranks the expected linear relationship is recovered, while lower ranks do not capture the final state sufficiently well to maintain this linearity. Note however that at weaker couplings results agree reasonably well between both $R=16$ and 128, and it is only at stronger couplings that one is required to use a larger rank to obtain accurate results.
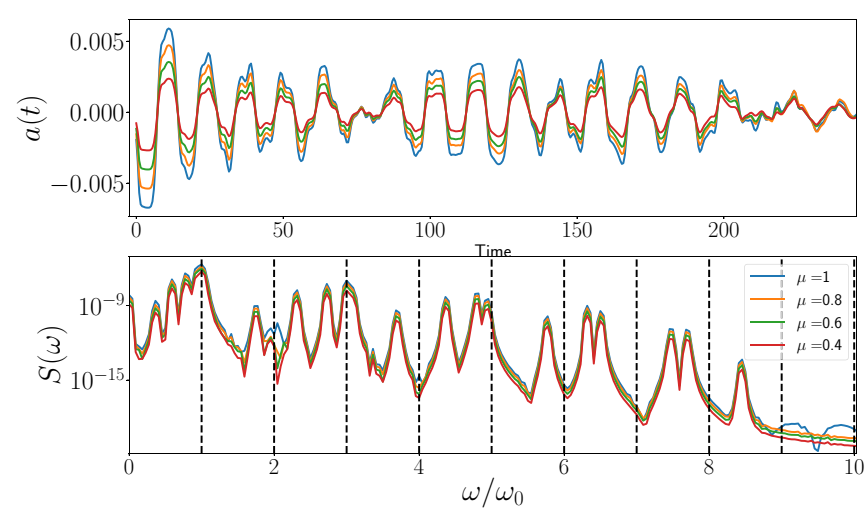

FIG. 8. Dipole acceleration and its frequency spectrum for a $U=0, N=14$ site system with a relatively strong coupling $\Gamma=$ $0.1 t_{0}$. One finds good convergence at $R=128$, with the spectrum displaying a number of prominent frequencies.

Figure 8 demonstrates that incoherent dissipative driving generates nonlinear effects in the transient dipole acceleration, analogously to the coherent external driving which produces HHG. In this instance, without an external driving frequency to compare to, we instead define a "fundamental frequency" $\omega_{0}$ as the most strongly represented frequency by taking $S(\omega)=|F F T\{a(t)\}|^{2}$ and setting

$$
\max _{\omega} S(\omega)=S\left(\omega_{0}\right)
$$

Examining the dipole acceleration spectrum, we find that the transient current contains number of distinct frequencies. This is particularly interesting when contrasted to the case of an isolated $U=0$ system driven by an external transformlimited pulse, where overtones only appear at odd integer multiples of the driving frequency [105]. In the present case, while we observe a prominent peak in the spectrum at the third harmonic, there are also a number of off-integer and sublinear frequencies present. The position of these peaks (including $\omega_{0}$ ) remain constant over a wide range of $\Gamma$ and $\mu$, indicating that while the transient system response to constant incoherent driving is nonlinear, the dissipative parameters meaningfully affect only the size of that response rather than its spectral character.

\section{DISCUSSION}

We have introduced an approximate method for the fast simulation of dissipative quantum systems. This technique is based upon first representing an infinitesimal Lindblad evolution in a Kraus form, from which one can construct an ensemble of wave functions which capture the full dynamics of the system. When the size of the ensemble exceeds a prespecified rank $R$, it is truncated in the space of its principal components. We have termed this method ensemble rank truncation (ERT), and have found a number of significant results. First, this method may employed in combination with matrix product state (MPS) wave-function decompositions, and under certain easily satisfiable conditions ERT + MPS will in principle be more efficient than either MPS alone or MPS + WMC in simulating large dissipative systems. 
The performance of ERT was investigated in a number of physically distinct systems, allowing for direct comparison to WMC simulations. Here it was found that in the regime of weak dissipative coupling, ERT offered order of magnitude improvements in both accuracy and computing time compared to WMC. This result is at least partially due to the difference between ERT and WMC in their rate of convergence to the true solution. In the latter case, WMC asymptotically converges at $O\left(\sqrt{n_{\text {traj }}}\right)$, while the convergence of ERT with $R$ will depend upon the specifics of the system dynamics. While one cannot determine a priori the appropriate rank to use with ERT, it should be possible to use system identification techniques [106-108] to ascertain the effective rank of the system before beginning an ERT simulation. As we have seen in all the cases considered, the rank $R$ required to accurately model a system with ERT is much lower than either the Hilbert space dimension $N_{H}$ or the number of trajectories $n_{\text {traj }}$ required by WMC.

An important question is how ERT will scale in parallel implementations using many processors. For WMC each trajectory is independent, and the speedup from multiprocessing will be approximately linear [88]. For ERT, each infinitesimal Kraus map may also be independently applied to each element of the ensemble. This part of the scheme is therefore trivially parallelizable and we would expect a similar linear scaling. Although the orthogonalization step requires some cross-communication to calculate the overlap matrix $S$, we found in Sec. II that the cost of this step compared to evolving an individual element of the ensemble will be small when $N_{H} \gg L^{2}$. We therefore expect that in this scenario, a properly parallelized ERT implementation will scale with number of processors approximately as well as WMC.
While the advantages of ERT have already been demonstrated, there remains a good deal of scope for improving the method. For instance, one might choose to adopt an adaptive rank $R$ at each step. As a simple example, one could improve accuracy by choosing $R$ at each step such that the sum of discarded eigenvalues in Eq. (20) is below some tolerance. Furthermore, it should be possible to improve on the $O\left(d t^{2}\right)$ error found in Eq. (6). This could be achieved either by rederiving $\hat{\mathcal{U}}_{k}, \hat{\mathcal{V}}_{k}$ using higher order Suzuki-Trotter decompositions [109], or through the application of Richardson extrapolation to Eq. (6). A final avenue for extension would be to improve the domain of applicability of the ERT, deriving the infinitesimal Kraus operators for the non-Markovian generalization of the Lindblad equation [110].

In summary, ERT is a novel technique for the simulation of dissipative quantum systems that is capable of outperforming existing techniques, and can in principle be alloyed to stateof-the-art methods for approximating large quantum systems.

\section{ACKNOWLEDGMENTS}

G.M. and D.I.B. are supported by Air Force Office of Scientific Research (AFOSR) (Grant No. FA9550-16-1-0254), Army Research Office (ARO) (Grant No. W911NF-19-10377), and Defense Advanced Research Projects Agency (DARPA) (Grant No. D19AP00043). The views and conclusions contained in this document are those of the authors and should not be interpreted as representing the official policies, either expressed or implied, of AFOSR, ARO, DARPA, or the U.S. Government. The U.S. Government is authorized to reproduce and distribute reprints for Government purposes notwithstanding any copyright notation herein.
[1] D. V. Zhdanov, D. I. Bondar, and T. Seideman, Friction as a consistent quantum-mechanical concept, Phys. Rev. A 98, 042133 (2018).

[2] S. L. Vuglar, D. V. Zhdanov, R. Cabrera, T. Seideman, C. Jarzynski, and D. I. Bondar, Nonconservative Forces Via Quantum Reservoir Engineering, Phys. Rev. Lett. 120, 230404 (2018).

[3] M. J. Kastoryano, F. Reiter, and A. S. Sørensen, Dissipative Preparation of Entanglement in Optical Cavities, Phys. Rev. Lett. 106, 090502 (2011).

[4] F. Reiter, D. Reeb, and A. S. Sørensen, Scalable Dissipative Preparation of Many-Body Entanglement, Phys. Rev. Lett. 117, 040501 (2016).

[5] R. Lescanne, S. Deléglise, E. Albertinale, U. Réglade, T. Capelle, E. Ivanov, T. Jacqmin, Z. Leghtas, and E. Flurin, Irreversible Qubit-Photon Coupling for The Detection of Itinerant Microwave Photons, Phys. Rev. X 10, 021038 (2020).

[6] A. Metelmann and A. A. Clerk, Nonreciprocal Photon Transmission and Amplification Via Reservoir Engineering, Phys. Rev. X 5, 021025 (2015).

[7] R. Schmidt, A. Negretti, J. Ankerhold, T. Calarco, and J. T. Stockburger, Optimal Control of Open Quantum Systems: Cooperative Effects of Driving and Dissipation, Phys. Rev. Lett. 107, 130404 (2011).
[8] C. S. DiLoreto and C. Rangan, Polarization control of spontaneous emission for rapid quantum-state initialization, Phys. Rev. A 95, 043834 (2017).

[9] A. Redfield, The theory of relaxation processes, in Advances in Magnetic Resonance, Advances in Magnetic and Optical Resonance, Vol. 1, edited by J. S. Waugh (Academic Press, 1965), pp. 1-32.

[10] A. G. Redfield, On the theory of relaxation processes, IBM J. Res. Dev. 1, 19 (1957).

[11] W. T. Pollard and R. A. Friesner, Solution of the redfield equation for the dissipative quantum dynamics of multilevel systems, J. Chem. Phys. 100, 5054 (1994).

[12] W. T. Pollard, A. K. Felts, and R. A. Friesner, The redfield equation in condensed-phase quantum dynamics, in Advances in Chemical Physics (Wiley, 2007), pp. 77-134.

[13] G. McCauley, B. Cruikshank, D. I. Bondar, and K. Jacobs, Accurate lindblad-form master equation for weakly damped quantum systems across all regimes, npj Quantum Inf. 6, 74 (2020).

[14] R. P. Feynman and F. L. Vernon, The theory of a general quantum system interacting with a linear dissipative system, Ann. Phys. 24, 118 (1963).

[15] G. McCaul and D. I. Bondar, How to win friends and influence functionals: Deducing stochasticity from deterministic dynamics, arXiv:1904.04918. 
[16] H. Kleinert, Path Integrals in Quantum Mechanics, Statistics, Polymer Physics and Financial Markets, 3rd ed. (World Scientific, Berlin, 2006).

[17] H. Kleinert and S. V. Shabanov, Quantum langevin equation from forward-backward path-integral, Phys. Lett. A 200, 224 (1995).

[18] K. Tsusaka, Generalized quantum Langevin equations from the forward-backward path integral, Phys. Rev. E 59, 4931 (1999).

[19] C. M. Smith and A. O. Caldeira, Generalized Feynman-Vernon approach to dissipative quantum systems, Phys. Rev. A 36, 3509 (1987).

[20] N. Makri, Effective non-oscillatory propagator for Feynman path integration in real time, Chem. Phys. Lett. 159, 489 (1989).

[21] K. Allinger and M. A. Ratner, Influence functionals: General methodology for subsystem calculations, Phys. Rev. A 39, 864 (1989).

[22] C. Bhadra and D. Banerjee, System-reservoir theory with anharmonic baths: A perturbative approach, J. Stat. Mech. (2016) 043404.

[23] H. K. McDowell, Quantum generalized Langevin equation: Explicit inclusion of nonlinear system dynamics, J. Chem. Phys. 112, 6971 (2000).

[24] A. Caldeira and A. Leggett, Path integral approach to quantum Brownian motion, Phys. A 121, 587 (1983).

[25] G. W. Ford and M. Kac, On the quantum langevin equation, J. Stat. Phys. 46, 803 (1987).

[26] C. W. Gardiner, Quantum noise and quantum Langevin equations, IBM J. Res. Develop. 32, 127 (1988).

[27] K. Sebastian, The classical description of scattering from a quantum system, Chem. Phys. Lett. 81, 14 (1981).

[28] A. Leggett, S. Chakravarty, A. Dorsey, M. Fisher, A. Garg, and W. Zwerger, Dynamics of the dissipative two-state system, Rev. Mod. Phys. 59, 1 (1987).

[29] N. G. van Kampen, Derivation of the quantum Langevin equation, J. Molec. Liquids 71, 97 (1997).

[30] P. P. Orth, A. Imambekov, and K. LeHur, Nonperturbative stochastic method for driven spin-boson model, Phys. Rev. B 87, 014305 (2013).

[31] P. P. Orth, A. Imambekov, and K. LeHur, Universality in dissipative Landau-Zener transitions, Phys. Rev. A 82, 032118 (2010).

[32] J. Ankerhold, P. Pechukas, and H. Grabert, Strong Friction Limit in Quantum Mechanics: The Quantum Smoluchowski Equation, Phys. Rev. Lett. 87, 086802 (2001).

[33] S. A. Maier and J. Ankerhold, Quantum smoluchowski equation: A systematic study, Phys. Rev. E 81, 021107 (2010).

[34] J. T. Stockburger and H. Grabert, Exact c-Number Representation Of Non-Markovian Quantum Dissipation, Phys. Rev. Lett. 88, 170407 (2002).

[35] J. T. Stockburger, Simulating spin-boson dynamics with stochastic Liouville-von Neumann equations, Chem. Phys. 296, 159 (2004).

[36] J. T. Stockburger and T. Motz, Thermodynamic deficiencies of some simple Lindblad operators: A diagnosis and a suggestion for a cure, Fortschritte der Phys. 65, 1600067 (2017).

[37] G. M. G. McCaul, C. D. Lorenz, and L. Kantorovich, Partition-free approach to open quantum systems in harmonic environments: An exact stochastic liouville equation, Phys. Rev. B 95, 125124 (2017).

[38] G. M. G. McCaul, C. D. Lorenz, and L. Kantorovich, Driving spin-boson models from equilibrium using exact quantum dynamics, Phys. Rev. B 97, 224310 (2018).

[39] K. Jacobs, Quantum Measurement Theory and its Applications (Cambridge University Press, Cambridge, 2014).

[40] C. Gardiner and P. Zoller, Quantum Noise (Springer, New York, 2010).

[41] H.-P. Breuer and F. Petruccione, The Theory of Open Quantum Systems (Oxford University Press, Oxford, 2007).

[42] G. Lindblad, On the generators of quantum dynamical semigroups, Commun. Math. Phys. 48, 119 (1976).

[43] V. Gorini, A. Kossakowski, and E. C. G. Sudarshan, Completely positive dynamical semigroups of $\mathrm{N}$-level systems, J. Math. Phys. 17, 821 (1976).

[44] D. Manzano, A short introduction to the lindblad master equation, AIP Adv. 10, 025106 (2020).

[45] R. Jones, J. A. Needham, I. Lesanovsky, F. Intravaia, and B. Olmos, Modified dipole-dipole interaction and dissipation in an atomic ensemble near surfaces, Phys. Rev. A 97, 053841 (2018).

[46] D. A. Lidar, I. L. Chuang, and K. B. Whaley, DecoherenceFree Subspaces for Quantum Computation, Phys. Rev. Lett. 81, 2594 (1998).

[47] J. Metz, M. Trupke, and A. Beige, Robust Entanglement Through Macroscopic Quantum Jumps, Phys. Rev. Lett. 97, 040503 (2006).

[48] M. Mohseni, P. Rebentrost, S. Lloyd, and A. Aspuru-Guzik, Environment-assisted quantum walks in photosynthetic energy transfer, J. Chem. Phys. 129, 174106 (2008).

[49] M. B. Plenio and S. F. Huelga, Dephasing-assisted transport: Quantum networks and biomolecules, New J. Phys. 10, 113019 (2008).

[50] B. Kraus, H. P. Büchler, S. Diehl, A. Kantian, A. Micheli, and P. Zoller, Preparation of entangled states by quantum Markov processes, Phys. Rev. A 78, 042307 (2008).

[51] S. Scali, J. Anders, and L. A. Correa, Local master equations bypass the secular approximation, arXiv:2009.11324.

[52] F. Nathan and M. S. Rudner, Universal lindblad equation for open quantum systems, Phys. Rev. B 102, 115109 (2020).

[53] G. McCauley, B. Cruikshank, S. Santra, and K. Jacobs, Ability of Markovian master equations to model quantum computers and other systems under broadband control, Phys. Rev. Research 2, 013049 (2020).

[54] M. Brenes, J. J. Mendoza-Arenas, A. Purkayastha, M. T. Mitchison, S. R. Clark, and J. Goold, Tensor-Network Method To Simulate Strongly Interacting Quantum Thermal Machines, Phys. Rev. X 10, 031040 (2020).

[55] F. Verstraete, J. J. García-Ripoll, and J. I. Cirac, Matrix Product Density Operators: Simulation of Finite-Temperature and Dissipative Systems, Phys. Rev. Lett. 93, 207204 (2004).

[56] M. Zwolak and G. Vidal, Mixed-State Dynamics In OneDimensional Quantum Lattice Systems: A Time-Dependent Superoperator Renormalization Algorithm, Phys. Rev. Lett. 93, 207205 (2004).

[57] I. A. Luchnikov, S. V. Vintskevich, H. Ouerdane, and S. N. Filippov, Simulation Complexity of Open Quantum Dynam- 
ics: Connection With Tensor Networks, Phys. Rev. Lett. 122, 160401 (2019).

[58] U. Schollwöck, The density-matrix renormalization group in the age of matrix product states, Ann. Phys. 326, 96 (2011), January 2011 Special Issue.

[59] A. Chenu, M. Beau, J. Cao, and A. del Campo, Quantum Simulation of Generic Many-Body Open System Dynamics Using Classical Noise, Phys. Rev. Lett. 118, 140403 (2017).

[60] M. D. Srinivas and E. B. Davies, Photon-counting probabilities in quantum optics, Opt. Acta 28, 981 (1981).

[61] N. Gisin, A Model For The Macroscopic Description And Continual Observations in Quantum Mechanics, Phys. Rev. Lett. 52, 1657 (1984).

[62] L. Diosi, Stochastic pure state representation for open quantum systems, Phys. Lett. A 114, 451 (1986).

[63] V. P. Belavkin, Non-demolition measurement and control in quantum dynamical systems, Information, complexity and control in quantum physics, in Proceedings of the 4th International Seminar on Mathematical Theory of Dynamical Systems and Microphysics, edited by A. Blaquiere, S. Diner, and G. Lochak (Springer-Verlag, New York, 1987), pp. 331-336.

[64] H. J. Carmichael, S. Singh, R. Vyas, and P. R. Rice, Photoelectron waiting times and atomic state reduction in resonance fluorescence, Phys. Rev. A 39, 1200 (1989).

[65] H. M. Wiseman and G. J. Milburn, Quantum theory of fieldquadrature measurements, Phys. Rev. A 47, 642 (1993).

[66] G. C. Hegerfeldt and T. S. Wilser, Ensemble or individual system, collapse or no collapse: A description of a single radiating atom, Classical and quantum systems, in Proceedings of the Second International Wigner Symposium, edited by H. D. Doebner, W. Scherer, and F. Schroeck (World Scientific, Singapore, 1992), pp. 104-115.

[67] K. Mølmer, Y. Castin, and J. Dalibard, Monte carlo wavefunction method in quantum optics, J. Opt. Soc. Am. B 10, 524 (1993).

[68] M. Wilde, Quantum Information Theory (Cambridge University Press, Cambridge, UK, 2013).

[69] K. Kraus, A. Böhm, J. D. Dollard, and W. H. Wootters (eds.), States, Effects, and Operations Fundamental Notions of Quantum Theory (Springer, Berlin, Heidelberg, 1983).

[70] R. Alicki, Quantum Dynamical Semigroups and Applications (Springer-Verlag, Berlin New York, 1987).

[71] D. M. Tong, J. L. Chen, J. Y. Huang, L. C. Kwek, and C. H. Oh, Kraus representation for the density operator of a qubit, Laser Phys. 16, 1512 (2006).

[72] M. Arsenijević, J. Jeknić-Dugić, and M. Dugić, Kraus operators for a pair of interacting qubits: A case study, Braz. J. Phys. 48, 242 (2018).

[73] R. Wu, A. Pechen, C. Brif, and H. Rabitz, Controllability of open quantum systems with Kraus-map dynamics, J. Phys. A: Math. Theor. 40, 5681 (2007).

[74] M. Nielsen, Quantum Computation and Quantum Information (Cambridge, University Press, Cambridge New York, 2010).

[75] H. Nakazato, Y. Hida, K. Yuasa, B. Militello, A. Napoli, and A. Messina, Solution of the lindblad equation in the kraus representation, Phys. Rev. A 74, 062113 (2006).

[76] K. M. Audenaert and S. Scheel, On random unitary channels, New J. Phys. 10, 023011 (2008).
[77] F. Casas, A. Murua, and M. Nadinic, Efficient computation of the Zassenhaus formula, Comput. Phys. Commun. 183, 2386 (2012).

[78] I. T. Jolliffe, Principal Component Analysis (Springer, New York, 2002).

[79] W. Wyss, Two non-commutative binomial theorems, arXiv:1707.03861.

[80] A. Hosseini and M. M. Karizaki, On the non-commutative newton binomial formula, arXiv:1902.10939.

[81] G. Vidal, Efficient Classical Simulation Of Slightly Entangled Quantum Computations, Phys. Rev. Lett. 91, 147902 (2003).

[82] G. Vidal, Efficient Simulation of One-Dimensional Quantum Many-Body Systems, Phys. Rev. Lett. 93, 040502 (2004).

[83] R. Orús, A practical introduction to tensor networks: Matrix product states and projected entangled pair states, Ann. Phys. 349, 117 (2014).

[84] S. Gammelmark and K. Mølmer, Interacting spins in a cavity: Finite-size effects and symmetry-breaking dynamics, Phys. Rev. A 85, 042114 (2012).

[85] R. Verresen, F. Pollmann, and R. Moessner, Quantum dynamics of the square-lattice heisenberg model, Phys. Rev. B 98, 155102 (2018).

[86] W. Heisenberg, Zur theorie des ferromagnetismus, Zeitschrift für Physik 49, 619 (1928).

[87] M. Žnidarič, T. Prosen, and P. Prelovšek, Many-body localization in the heisenberg $x x z$ magnet in a random field, Phys. Rev. B 77, 064426 (2008).

[88] J. R. Johansson, P. D. Nation, and F. Nori, QuTiP: An opensource Python framework for the dynamics of open quantum systems, Comput. Phys. Commun. 183, 1760 (2012).

[89] J. R. Johansson, P. D. Nation, and F. Nori, QuTiP 2: A Python framework for the dynamics of open quantum systems, Comput. Phys. Commun. 184, 1234 (2013).

[90] P. Solano, P. Barberis-Blostein, F. K. Fatemi, L. A. Orozco, and S. L. Rolsto, Super-radiance reveals infinite-range dipole interactions through a nanofiber, Nat. Commun. 8, 1857 (2017).

[91] J. S. Hodges, N. Y. Yao, D. Maclaurin, C. Rastogi, M. D. Lukin, and D. Englund, Timekeeping with electron spin states in diamond, Phys. Rev. A 87, 032118 (2013).

[92] T. Zhong, J. M. Kindem, J. G. Bartholomew, J. Rochman, I. Craiciu, V. Verma, S. W. Nam, F. Marsili, M. D. Shaw, A. D. Beyer, and A. Faraon, Optically Addressing Single Rare-Earth Ions in A Nanophotonic Cavity, Phys. Rev. Lett. 121, 183603 (2018).

[93] H. M. Wiseman and G. J. Milburn, Quantum Measurement and Control (Cambridge Press, Cambridge UK, 2010).

[94] H. Tasaki, The Hubbard model - An introduction and selected rigorous results, J. Phys. Cond. Mat. 10, 4353 (1998).

[95] G. McCaul, C. Orthodoxou, K. Jacobs, G. H. Booth, and D. I. Bondar, Controlling arbitrary observables in correlated manybody systems, Phys. Rev. A 101, 053408 (2020).

[96] G. McCaul, C. Orthodoxou, K. Jacobs, G. H. Booth, and D. I. Bondar, Driven Imposters: Controlling Expectations In ManyBody Systems, Phys. Rev. Lett. 124, 183201 (2020).

[97] K. Sponselee, L. Freystatzky, B. Abeln, M. Diem, B. Hundt, A. Kochanke, T. Ponath, B. Santra, L. Mathey, K. Sengstock, and C. Becker, Dynamics of ultracold quantum gases in the 
dissipative Fermi-Hubbard model, Quantum Sci. Technol. 4, 014002 (2019).

[98] M. Nakagawa, N. Tsuji, N. Kawakami, and M. Ueda, Dynamical Sign Reversal of Magnetic Correlations in Dissipative Hubbard Models, Phys. Rev. Lett. 124, 147203 (2020).

[99] P. Weinberg and M. Bukov, QuSpin: A Python package for dynamics and exact diagonalisation of quantum many body systems. Part II: Bosons, fermions and higher spins, SciPost Phys. 7, 20 (2019).

[100] P. Weinberg and M. Bukov, QuSpin: A Python package for dynamics and exact diagonalisation of quantum many body systems part I: Spin chains, SciPost Phys. 2, 003 (2017).

[101] S. Ghimire, A. D. DiChiara, E. Sistrunk, G. Ndabashimiye, U. B. Szafruga, A. Mohammad, P. Agostini, L. F. DiMauro, and D. A. Reis, Generation and propagation of high-order harmonics in crystals, Phys. Rev. A 85, 043836 (2012).

[102] S. Ghimire, A. D. DiChiara, E. Sistrunk, P. Agostini, L. F. DiMauro, and D. A. Reis, Observation of high-order harmonic generation in a bulk crystal, Nat. Phys. 7, 138 (2011).

[103] Y. Murakami, M. Eckstein, and P. Werner, High-Harmonic Generation In Mott Insulators, Phys. Rev. Lett. 121, 057405 (2018).
[104] T. Prosen and M. Žnidarič, Diffusive high-temperature transport in the one-dimensional hubbard model, Phys. Rev. B 86, 125118 (2012).

[105] R. E. Silva, I. V. Blinov, A. N. Rubtsov, O. Smirnova, and M. Ivanov, High-harmonic spectroscopy of ultrafast many-body dynamics in strongly correlated systems, Nat. Photonics 12, 266 (2018).

[106] C. H. Lee and J. N. Juang, Deterministic bilinear system identification, J. Astronaut. Sci. 60, 237 (2013).

[107] J. W. Van Wingerden and M. Verhaegen, Subspace identification of bilinear systems using a dedicated input sequence, in Proceedings of the IEEE Conference on Decision and Control, 4962 (IEEE, Piscataway, NJ, 2007).

[108] J. N. Juang, Continuous-time bilinear system identification, Nonlinear Dyn. 39, 79 (2005).

[109] N. Hatano and M. Suzuki, Finding exponential product formulas of higher orders, in Quantum Annealing and Other Optimization Methods (Springer, Berlin, Heidelberg, 2005), pp. 37-68.

[110] H.-P. Breuer, Non-markovian generalization of the lindblad theory of open quantum systems, Phys. Rev. A 75, 022103 (2007). 